\title{
Adaptación transcultural y validación de un cuestionario de cuidado humanizado en enfermería para una muestra de población Chilena
}

\author{
Transcultural adaptation and validation of a humanized-care questionnaire in \\ nursing for a Chilean population sample
}

\author{
Adaptação transcultural e validação de um questionário de cuidado humanizado \\ em enfermagem para uma amostra da população Chilena
}

\author{
Angélica Melita Rodríguez ${ }^{1}{ }^{\oplus}$, Patricia Jara Concha ${ }^{2}{ }^{\circledR}$, Daniel Ignacio Pereira ${ }^{3}$, Luis Luengo Machuca ${ }^{4}$
}

\section{Histórico}

Recibido:

23 de febrero de 2018

Aceptado:

19 de abril de 2018

1 Candidata a Doctora en Enfermería. Académica Universidad de Concepción. Concepción, Chile.

2 Doctora en Enfermería. Académica Universidad de Concepción. Concepción, Chile. Autor de Correspondencia. E-mail:pjara@udec.cl

3 Doctor en Lingüistica. Académico Universidad de Concepción. Concepción, Chile.

4 Magister en Estadística Aplicadar Académico Universidad de Concepción. Concepción, Chile Introducción: La humanización del cuidado es una temática relevante de tratar por el impacto que tiene en la salud de las personas, esto demanda contar con instrumentos confiables y válidos que permitan su evaluación. Objetivo: Adaptar y validar la versión colombiana del cuestionario de Percepción de Comportamientos de Cuidados Humanizados en Enfermería (PCHE), para ser utilizado en población chilena. Materiales y Métodos: Estudio de validación, que consistió en la evaluación por expertos y adaptación transcultural del PCHE a través de un enfoque intralingual y el análisis de la consistencia interna y validez de constructo del instrumento PCHE versión Chilena. Resultados: Se modificaron términos y tiempos verbales, con un $100 \%$ de comprensión por parte de los encuestados, esto permitió generar un instrumento adecuado al lenguaje y comprensión del español utilizado en Chile. Se obtuvo un alto coeficiente de alfa de Cronbach de 0.92, y una validez de constructo por convergencia con una alta correlación positiva r de 0,73 con la Escala de Percepción del Cuidado Humanizado. Discusión: Se visualiza la importancia de la adaptación lingüística sociocultural de instrumentos en distintas culturas y de esta forma evitar sesgos derivados del lenguaje. La confiabilidad y validez de constructo del instrumento es similar a la encontrada en Colombia, lo que demuestra las adecuadas propiedades y su buen funcionamiento en un contexto cultural distinto. Conclusiones: Este instrumento constituye un aporte a la humanización de los cuidados en enfermería, al ser una escala válida y apropiada para ser usada en el contexto de la población Chilena.

Palabras clave: Estudios de Validación; Lingüística; Encuestas y Cuestionarios; Humanización de la Atención; Características Culturales.

Introduction: Humanization of care is a relevant theme to address due to its impact on the health of individuals; this requires having reliable and valid instruments that permit its evaluation. Objective: Adapt and validate the Colombian version of the questionnaire on Perception of Humanized-Care Behaviors in Nursing (PHCBN) for use in a Chilean population. Materials and Methods: Validation study consisting in the expert evaluation and transcultural adaptation of the PHCBN through an intra-lingual approach and internal consistency analysis and construct validity of the Chilean version of the instrument. Results: Terms and verb tenses were modified with $100 \%$ comprehension by those surveyed; this permitted generating an instrument suited to the language and comprehension of the Spanish used in Chile. The study obtained a high Cronbach's alpha coefficient of 0.92 , along with a construct validity through convergence with a high positive $r$ correlation of 0.73 with the Humanized-Care Perception Scale. Discussion: Herein, we visualize the importance of the linguistic sociocultural adaptation of instruments in different cultures and, thus, avoid bias derived from language. The reliability and construct validity of the instrument is similar to that found in Colombia, which demonstrates adequate properties and its good function within a distinct cultural context. Conclusions: This instrument constitutes a contribution to the humanization of nursing care, given that it is a valid and appropriate scale for use in the Chilean population context.

Key words: Validation Studies; Linguistics; Surveys and Questionnaires; Humanization of Assistance; Cultural Characteristics.

Resumo

Introdução: A humanização do cuidado é uma questão relevante, abordada pelo impacto que tem na saúde das pessoas, o que requer disponibilizar de instrumentos confiáveis e válidos que permitam sua avaliação. Objetivo: Adaptar e validar a versão Colombiana do questionário de Percepção de Comportamentos de Cuidados Humanizados em Enfermagem (PCHE), para ser utilizado em populacão Chilena. Materiais e Métodos: Estudo de validacão, que consistiu na avaliação de peritos e adaptação transcultural do PCHE através de uma abordagem intralinguística e a análise da consistência interna e validade de construto do instrumento PCHE versão Chilena. Resultados: Foram modificados termos e tempos verbais, com $100 \%$ de compreensão por parte dos entrevistados, isto permitiu gerar um instrumento adequado para a linguagem e compreensão do espanhol utilizado no Chile. Obteve-se um elevado coeficiente alfa de Cronbach de 0,92 , e validade de construto por convergência com uma alta correlação positiva $r$ de 0,73 com a Escala de Percepcão do Cuidado Humanizado. Discussão: Visualiza-se a importância da adaptação linguística sociocultural de instrumentos em diferentes culturas e, assim, evitar vieses derivados da linguagem. A confiabilidade e a validade de constructo do instrumento são semelhantes às encontradas na Colômbia, o qual demostra as adequadas propriedades e seu bom funcionamento em um contexto cultural diferente. Conclusões: Este instrumento constitui uma contribuição para a humanização dos cuidados em enfermagem, por ser uma escala válida e apropriada para ser utilizada no contexto da população Chilena.

Palavras chave: Estudos de Validação; Linguística; Inquéritos e Questionários; Humanização da Assistência; Características Culturais.

Como citar este artículo: Rodríguez AM, Jara P, Pereira DI, Luengo L. Adaptación transcultural y validación de un cuestionario de cuidado humanizado en enfermería para una muestra de población Chilena. Rev Cuid. 2018; 9(2): 2245-56. http://dx.doi.org/10.15649/cuidarte.v9i2.531

(c) (1) (9) 2018 Universidad de Santander. Este es un artículo de acceso abierto, distribuido bajo los términos de la licencia Creative Commons Attribution (CC BY-NC 4.0), que permite el uso ilimitado, distribución y reproducción en cualquier medio, siempre que el autor original y la fuente sean debidamente citados. 


\section{INTRODUCCIÓN}

En la actualidad, se puede constatar que múltiples factores han distanciado la relación entre los trabajadores y las personas usuarias de los sistemas de salud ${ }^{1,2}$, razón por la cual la humanización en este ámbito, se ha trasformado en una demanda creciente.

Múltiples investigaciones evidencian a pacientes que declaran que quienes deben estar más cercanos a ellos en circunstancias críticas, como en el proceso de enfermedad, están más centrados en técnicas y procedimientos que finalmente los alejan de ese cuidado integral, cercano y más humano ${ }^{3-5}$. Para enfermería, esta temática se ha trasformado en un foco de interés, por el impacto que la humanización del cuidado tiene en el proceso de recuperación de las personas. Así lo confirman estudios de índole cualitativo, que profundizan principalmente en cómo vivencian la humanización del cuidado las personas bajo diversas circunstancias de salud ${ }^{6-8}$. Al ser este un aspecto tan relevante en la entrega de cuidado de enfermería y el cual repercute directamente en la calidad de la atención y la satisfacción de los pacientes $^{9}$, se visualiza la necesidad de contar con instrumentos que permitan monitorizar $\mathrm{y}$ evaluar el cuidado desde esta perspectiva más humana. Jean Watson, una de las principales exponentes de la humanización del cuidado en el ámbito de la enfermería, proporciona el marco para la elaboración de diversos instrumentos, en un afán de evaluar y promover la humanización de los cuidados ${ }^{10-12}$, éstos elaborados bajo un prisma cultural norteamericano $\mathrm{y}$ en lengua inglesa, han sido validados lingüística, cultural y psicométricamente para poder ser utilizados de manera confiable en la población latinoamericana ${ }^{13,14}$. En Colombia, uno de los países que más ha abordado esta temática, se han creado otros instrumentos basados en los planteamientos de Watson ${ }^{15,16}$, los cuales, si bien han demostrado ser confiables y válidos para ser utilizados en población colombiana, no se puede asegurar que estos sean aplicables en otros grupos humanos con características socioculturales diferentes, ya que si bien en América Latina se habla mayoritariamente la lengua española, esta adquiere formas propias de manifestarse que varían entre las distintas comunidades hablantes, influenciadas por aspectos propios de la cultura local ${ }^{17,18}$. Por este motivo surge el interés de desarrollar este trabajo, ya que para obtener resultados que reflejen rigurosidad científica y permitan hacer reales comparaciones con otros estudios latinoamericanos, se hace necesario evitar todo tipo de sesgos, entre ellos los derivados del lenguaje, que pudieran interferir en el proceso de comunicación y obtención de información, permitiendo la correcta utilización e interpretación del cuestionario tanto por parte del investigador como del encuestado. En este contexto, en el año 2001 en Colombia, bajo la autoría de Rivera y Triana $^{19}$, se crea el instrumento denominado: "Percepción de los Comportamientos de Cuidado Humanizado en Enfermería (PCHE)". En su primera versión, dicho instrumento constó de 50 items ${ }^{19}$. En el año 2008, Reina y $\operatorname{Vargas}^{15}$, lo someten a validación de contenido y facial, lo que dio como resultado 
un cuestionario válido y confiable para ser aplicado $^{15}$.

Posteriormente, en el año 2014, Gonzalez ${ }^{16}$, basándose en la experiencia anterior, procede a realizar ajustes a 12 ítems, de manera que fueran más claros para los sujetos de aplicación, obteniendo una validez facial de 0,92 y un índice de validez de contenido de 0,98. El Alfa de Cronbach calculado para esta nueva versión del instrumento fue de 0,96. Finalmente, el test quedó constituido por 32 preguntas en formato de escala Likert de cuatro opciones: siempre, casi siempre, algunas veces y nunca. Las preguntas tributan a tres grandes categorías: cualidades del hacer de enfermería, apertura a la comunicación enfermera(o)-paciente y disposición para la atención ${ }^{16}$.

Dado el alto grado de utilidad del instrumento PCHE para medir cuidados humanizados en enfermería, la investigación que aquí se presenta tiene como propósito adaptar y validar la versión colombiana del instrumento PCHE, versión 2014, para ser aplicado en población chilena, de manera que responda de mejor modo a las diferencias socioculturales y lingüísticas existentes entre ambas comunidades de destino, para así evitar sesgos derivados del lenguaje ${ }^{17,18}$.

\section{MATERIALES Y MÉTODOS}

Tipo de estudio: Este corresponde a un estudio de validación de un cuestionario desarrollado en dos fases. En la primera se realizó la adaptación transcultural, en la fase dos, se procedió a evaluar la consistencia interna y validez de constructo del instrumento PCHE versión español-chileno.

Período del estudio: Este trabajo fue desarrollado durante el primer semestre del año 2017.

\section{Población de estudio y tamaño de muestra:} Para la primera fase del estudio, adaptación sociolingüística, la población correspondió a personas de nacionalidad chilena de la Comuna de Concepción, no institucionalizadas, de diferente sexo, nivel de instrucción y distribuidos en tres segmentos etarios (jóvenes, adultos y adultos mayores) basados en la experiencia de Torres et $\mathrm{al}^{20}$, con el objetivo de obtener un resultado que integrara la visión de diversos grupos humanos ${ }^{20}$. Por tanto, se aplicó el instrumento "PCHE 2014 versión colombiana"16, a una muestra aleatoria estratificada de 60 personas.

Para la segunda fase de la investigación, dirigida a determinar la validez y fiabilidad del instrumento PCHE versión español-chileno, se procedió a aplicarlo a una muestra aleatoria simple de 19 pacientes hospitalizados del Servicio de Especialidades de un de un Hospital dependiente del Servicio de Salud Talcahuano, quienes formaron parte de la prueba piloto del instrumento.

\section{Procedimientos y recolección de datos:}

En la primera fase se realizó la adaptación sociolingüística, como no existe consenso sobre la metodología que debe seguirse para adaptar 
un instrumento a otro contexto cultural que comparten el idioma de la versión original o de una de sus versiones adaptadas, se trabajó con un enfoque intralingual, es decir, de adaptación en la misma lengua, la que contempla una adaptación lingüística de ajuste ${ }^{21}$. Para tal efecto, se utilizó el método de análisis lingüístico por ítem, que consistió en buscar equivalencias sociopragmáticas para los ítems del cuestionario, considerando las diferencias culturales entre las comunidades de origen y destino, con el objetivo de identificar términos de uso poco frecuente o con un significado distinto al del lugar donde se pretende aplicar el instrumento ${ }^{22,23}$, de esta forma se procedió a sustituir esos vocablos por otros de mejor comprensión para el lenguaje utilizado en Chile. Este proceso de ajuste de contenido de los ítems del instrumento se hace necesario para adaptarlo a la visión particular de la comunidad de destino, de modo que el resultado estuviera provisto de "viabilidad cultural" 24 , dando como resultado una equivalencia lingüística entre ambas versiones, que se desarrolló como se explica a continuación:

Aplicación del instrumento "PCHE 2014 versión colombiana"16, a una muestra aleatoria estratificada de 60 personas a quienes se les solicitó que lo respondieran, para evaluar dos criterios: claridad y comprensión, a su vez, el cuestionario tenía adosada una matriz de registro de comentarios, observaciones y sugerencias permitiendo pesquisar la presencia de dificultades en la comprensión del instrumento por parte del encuestado. Posteriormente, los resultados de esta etapa fueron revisados y analizados en conjunto con un experto en lingüística, cambiando vocablos y términos que facilitaran la claridad y comprensión de los enunciados para el lector. Es así como se logró adecuar el instrumento original a la cultura chilena.

Para la fase dos del estudio, la confiabilidad se analizó a través de la consistencia interna utilizando el coeficiente de Alfa de Cronbach, para este estudió se consideró que los reactivos medían en forma óptima el constructo cuando los valores encontrados estuvieran entre $0,7 \mathrm{y}$ $0,9^{25}$. La validez del instrumento se analizó a través de validez de constructo ${ }^{26}$, para tal efecto se utilizó un método correlacional, aplicando un test que midiera los mismos aspectos o muy similares contemplados en el cuestionario PCHE adaptado lingüísticamente para población chilena, lo cual se denomina validación por convergencia o convergente, la cual indica las correlaciones positivas con otro test que mida lo mismo $^{27}$. Para cumplir con este criterio se utilizó el cuestionario: "Escala de evaluación de la percepción del cuidado humanizado brindado por el profesional de enfermería" $(\mathrm{ECH})$ de Hermosilla et $\mathrm{al}^{28}$, validado para población chilena, el cual está compuesto por 36 ítems o reactivos $^{28}$, instrumentos que fueron aplicados de manera simultánea a un mismo grupo de pacientes.

Variables del estudio: En primera fase del estudio se manejaron como variables la claridad y comprensión del instrumento "Percepción de los comportamientos de cuidado humanizado en enfermería". 
Para la fase de dos se consideraron como variables la percepción de los comportamientos de cuidado humanizado en enfermería, más calidad, comunicación y disposición; para determinar la validez y confiabilidad del instrumento.

Análisis de datos: En la primera fase del estudio se analizó la información en conjunto con el experto en lingüística, procediendo a reemplazar algunos vocablos no habituales en el léxico básico del español de $\mathrm{Chile}^{29}$, por otros más frecuentes, de acuerdo con la Lista de Frecuencias de Palabras del Castellano de Chile (Lifcach) ${ }^{30}$, lo cual facilitara la claridad y comprensión del instrumento en la población chilena.

Para la fase dos de la investigación se procedió al análisis de datos, utilizando el coeficiente alfa de Cronbach para cuantificar consistencia interna; análisis de normalidad con medidas de resumen y prueba de Shapiro-Wilk. Para el análisis de convergencia, se calculó el coeficiente de correlación de Spearman. Se consideró significancia del 5\% y el software utilizado fue SPSS versión 23.

Consideraciones éticas: Para el desarrollo de este estudio se procedió a solicitar autorización de los autores originales del PCHE, Nelly Rivera y Álvaro Triana, y a Óscar González, autor de la última versión validada para Colombia. Estas fueron otorgadas mediante la firma de un acuerdo de recepción y uso de instrumento. El proyecto de investigación fue presentado y aprobado en el Comité de Ética de la Facultad de Enfermería de la Universidad de Concepción con resolución $n^{\circ}$ 014-16 y en el Comité Ético Científico del Servicio de Salud Talcahuano con acta de aprobación $\mathrm{N}^{\circ} 49$, entidad a la que pertenece el hospital donde se aplicó la investigación.

\section{RESULTADOS}

\section{Los resultados de este estudio se presentan en dos fases}

De la fase de adaptación sociocultural, cerca del $80 \%$ de los encuestados manifestaron comprender el cuestionario; del porcentaje restante, principalmente adultos mayores de nivel sociocultural bajo (usuarios frecuentes del sistema de salud público en Chile), solicitaron explicación de algunos de los ítems, ya que no lograban la comprensión absoluta de éste. Tras el análisis de esta información y en conjunto con el experto en lingüística, se procedió a reemplazar algunos vocablos no habituales en el léxico básico del español de $\mathrm{Chile}^{29}$, por otros más frecuentes, de acuerdo con la Lista de Frecuencias de Palabras del Castellano de Chile $(\text { Lifcach })^{30}$, obteniendo los siguientes resultados:

- El enunciado: "Le administran a tiempo los medicamentos formulados por el médico"; el verbo "administrar" fue sustituido por "dar" y el participio "formulados", por "indicados". De este modo, el ítem final fue: "Le dieron a tiempo los medicamentos indicados".

- Se modificó el tiempo verbal de todas las preguntas de los ítems, del presente indicativo 
al pretérito indefinido, pues el instrumento se aplica al paciente una vez finalizados los cuidados de enfermería propios de su tratamiento. De este modo, por ejemplo, el enunciado: "Le tratan con amabilidad"; fue reemplazado por: "Lo (la) trataron con amabilidad".

- En algunos ítems del instrumento original se observó el uso leísta del pronombre acusativo. Como no se trata de un fenómeno extendido en Chile $^{31}$, y que podría resultar extraño para algunos pacientes, se modificó a una versión no leísta y se agregó la forma femenina del pronombre. Por ejemplo, el enunciado: "Le llaman por su nombre"; se modificó por: "Lo (la) llamaron por su nombre". En algunos casos, el uso leísta fue reemplazado por construcciones reflexivas transitivas de "se" determinado efectivo ${ }^{32}$, como en "Le respetan sus decisiones", que fue cambiado por: "Se mostraron respetuosos por sus decisiones".

- A su vez, para hacer más cercano el instrumento, se insertó antes de los ítems el encabezado enmarcante: "Estimado(a) señor(a): A continuación, presentamos una serie de afirmaciones que describen los comportamientos de cuidado de enfermeras y enfermeros". Al final de todo, se agregó la frase de agradecimiento "Le agradecemos por haber respondido esta encuesta", de modo de incorporar elementos de cortesía verbal que otorgaran un efecto de disminución del esfuerzo ${ }^{33}$, que el paciente tuvo que hacer al contestar la encuesta.
Con estas modificaciones se procedió a la aplicación del instrumento, adaptado al lenguaje chileno, a una muestra de 19 pacientes hospitalizados del Servicio de Especialidades de un Hospital dependiente del Servicio de Salud Talcahuano, Chile. Los resultados de esta etapa evidenciaron un $100 \%$ de comprensión por parte de los encuestados, quienes fácilmente comprendían los enunciados y daban respuesta a las preguntas.

De la segunda fase de la investigación, se desprende el siguiente análisis descriptivo del cuestionario "PCHE transculturalmente adaptado a población chilena". Como se aprecia en la Tabla 1, se evidencian los siguientes aspectos: el puntaje mínimo con el cual se calificó la percepción de cuidado humanizado de enfermería fue de 83, lo cual corresponde al concepto "nunca" (nunca percibieron comportamientos de cuidado humanizado por parte de enfermería), a su vez la calificación máxima fue de 128, la cual corresponde al puntaje total de la escala, puntaje que a su vez está contemplado en el concepto de "siempre" (siempre percibió comportamientos de humanización en los cuidados entregados por enfermería), con un rango de 45 entre ambos puntajes. En relación a los valores obtenidos de media y mediana (112 y 119, respectivamente), se puede apreciar que la mayoría de las personas refirieron percibir "casi siempre" una percepción de comportamientos de cuidados humanizados brindados por enfermería. 
Tabla 1. Estadísticos descriptivos del cuestionario: Percepción de comportamiento de cuidado humanizado de enfermería. Unidad de Especialidades, Talcahuano, Chile, 2017

\begin{tabular}{|c|c|c|c|}
\hline \multirow[t]{13}{*}{ Total PCHE } & \multicolumn{2}{|l|}{ Media } & 112,52 \\
\hline & \multirow{2}{*}{$\begin{array}{l}95 \% \text { de intervalo de } \\
\text { confianza para la media }\end{array}$} & Límite inferior & 105,74 \\
\hline & & Límite superior & 119,30 \\
\hline & \multicolumn{2}{|l|}{ Media recortada al 5\% } & 113,30 \\
\hline & \multicolumn{2}{|l|}{ Mediana } & 119,00 \\
\hline & \multicolumn{2}{|l|}{ Varianza } & 198,04 \\
\hline & \multicolumn{2}{|l|}{ Desviación estándar } & 14,07 \\
\hline & \multicolumn{2}{|l|}{ Mínimo } & 83,00 \\
\hline & \multicolumn{2}{|l|}{ Máximo } & 128,00 \\
\hline & \multicolumn{2}{|l|}{ Rango } & 45,00 \\
\hline & \multicolumn{2}{|l|}{ Rango intercuartil } & 27,00 \\
\hline & \multicolumn{2}{|l|}{ Asimetría } &,- 67 \\
\hline & \multicolumn{2}{|l|}{ Curtosis } &,- 78 \\
\hline
\end{tabular}

PCHE: Escala de Percepción Comportamientos de Cuidado Humanizado en Enfermería.

Fuente: "Adaptación transcultural y validación de un cuestionario de cuidado humanizado en enfermería para una muestra de población chilena”. 2017.

En relación a la confiabilidad del instrumento, el coeficiente alfa de Cronbach resultó con un valor de 0.92 , lo cual evidencia que los elementos de la escala son homogéneos $y$, por lo tanto, que esta mide de forma consistente la propiedad para la cual fue diseñada (alta consistencia interna). Se concluye que este valor obtenido califica como aceptable la confiablidad del instrumento PCHE lingüísticamente adaptado para población chilena $^{25}$.

Para la validación de constructo, en primer lugar, se procedió a analizar normalidad de las escalas, para ello se aplicó la prueba ShapiroWilk obteniendo $\mathrm{p}=0,040$ y $\mathrm{p}=0,056$ para los instrumentos "PCHEadaptado transculturalmente a población chilena" y la "Escala de Evaluación de la Percepción del Cuidado Humanizado brindado por el profesional de enfermería $(\mathrm{ECH})$ " de Hermosilla et $\mathrm{al}^{28}$, respectivamente, rechazando normalidad, motivo por el cual se trabajó con la prueba de Rho de Spearman, la que arrojó un valor de 0.73 , evidenciando una correlación positiva entre ambos instrumentos, es decir, que ambos presentaron una convergencia positiva.

A su vez, al correlacionar las tres dimensiones del instrumento "PCHE adaptado transculturalmente a población chilena" (cualidades del hacer de enfermería [calidad], apertura a la comunicación enfermera(o) - paciente [comunicación] y disposición para atención [disposición]) con el puntaje total de la "Escala evaluación de la percepción de cuidado humanizado brindado por el profesional de enfermería (CHE)", como se muestra en la Tabla 2, evidenció una alta correlación positiva, entre todas las dimensiones y el puntaje total de esta escala. 
Tabla 2. Correlaciones entre dimensiones del instrumento "PCHE transculturalmente adecuado para población chilena" y la "Escala de evaluación de la percepción del cuidado humanizado brindado por el profesional de enfermería (ECH)". Unidad de Especialidades, Talcahuano, Chile, 2017

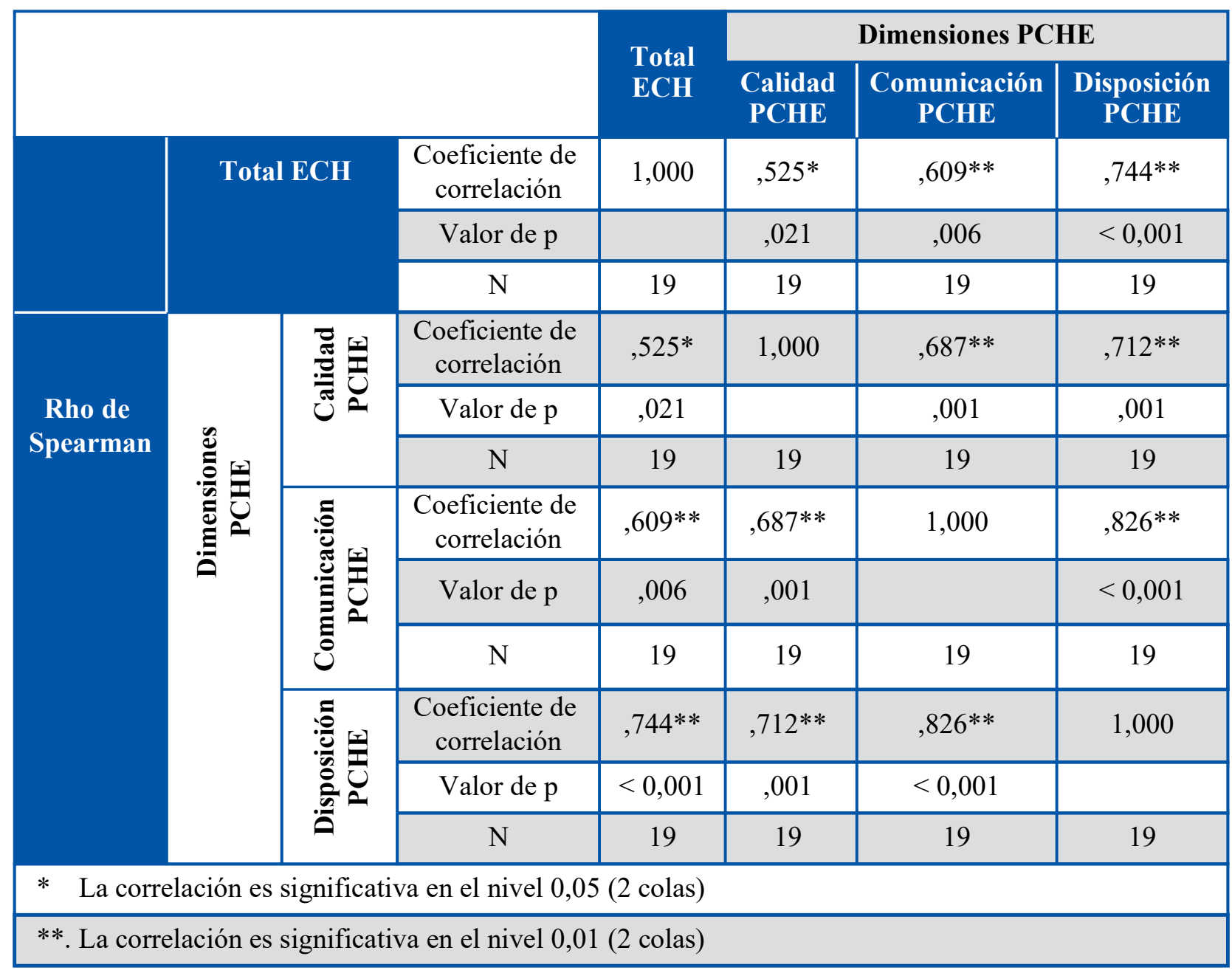

PCHE: Escala de Percepción Comportamientos de Cuidado Humanizado en Enfermería; ECH: Escala de evaluación de la percepción del cuidado humanizado brindado por el profesional de enfermería.

Fuente: "Adaptación transcultural y validación de un cuestionario de cuidado humanizado en enfermería para una muestra de población chilena”. 2017.

A través de estos resultados es posible confirmar la validez de constructo del instrumento "PCHE versión transculturalmente adaptada para población chilena", al presentar una alta correlación positiva con el instrumento $\mathrm{CHE}$, que mide criterios semejantes a los contemplados en el cuestionario sometido a evaluación, por lo cual se establece una validez de constructo positiva por convergencia.

\section{DISCUSIÓN}

La humanización del cuidado adquiere gran importancia para la práctica de enfermería, al entregar herramientas que permiten a la enfermera(o) otorgar un cuidado integral, basado en el respeto de la dignidad humana y de calidad, lo cual a su vez se ajusta con las normativas existentes en los distintos sistemas 
de salud ${ }^{34-37}$. En este contexto, surge el objetivo principal del presente estudio, el cual fue adaptar sociolingüísticamente y validar un instrumento de cuidado humanizado para ser aplicado en población chilena, para tal efecto se trabajó con el instrumento: Percepción de los comportamientos de cuidado humanizado en enfermería (PCHE) de los autores Rivera y Triana ${ }^{19}$. El instrumento PCHE versión Colombia, ha sido un cuestionario ampliamente utilizado en este país, sin embargo según lo planteado por expertos, a pesar de compartir el mismo idioma, existen diferencia socioculturales plasmadas en el lenguaje y la forma de expresarse que en el ámbito de la investigación pueden influir profundamente, generando sesgos en el momento de la interpretación de resultados ${ }^{38}$. Esta realidad está presente en Latinoamérica, en donde en diversas zonas se comparte el idioma español, sin embargo la cultura influye profundamente en el lenguaje, lo cual puede impactar en la correcta comprensión de este ${ }^{21-23}$. Para que esta característica no afectara los resultados de la investigación, se sometió este instrumento a una adaptación sociocultural y una posterior validación, como lo plantea Barbero et $\mathrm{al}^{39}$, donde menciona la importancia de la calidad científica de test e instrumentos y que éstos, aunque hayan sido validados en un país, no pueden ser directamente utilizados en otro sin ser sometidos a un proceso de adaptación sociocultural y posterior validación, con lo cual se asegura su correcta utilización ${ }^{39}$.

Es así como en este estudio ciertos términos, verbos y enunciados, debieron ser modificados para facilitar esa comprensión de los encuestados chilenos, investigaciones similares han demostrado la importancia de modificar ciertos vocablos para la adecuada comprensión de lo que realmente se desea consultar y conocer ${ }^{22,23,40}$, así lo demuestran estudios como los de Rivero et $\mathrm{al}^{22}$. Y Ferreira et $\mathrm{al}^{40}$, en donde se hace énfasis en la adaptación sociocultural de los instrumentos para su adecuada comprensión e interpretación ${ }^{22,40}$. De esta forma, la validación del instrumento adaptado sociolingüísticamente para Chile, permitió corroborar las adecuadas características del cuestionario para ser utilizado en población chilena; en relación a la confiabilidad demostró una buena consistencia interna evidenciada por un alto coeficiente alfa de Cronbach, resultado similar al logrado por el instrumento colombiano en la validación de la versión $2014^{16}$. Además, el instrumento chileno presentó una alta correlación positiva con otro test que mide aspectos similares ${ }^{28}$, lo que permitió comprobar su validez convergente positiva y, por lo tanto, una adecuada validez de constructo. Esto constituye una novedad y un aporte del presente estudio, pues esta prueba no había sido aplicada en estudios anteriores y solo se habían evaluado otros ámbitos del instrumento ${ }^{15,16,19,41}$. En este proceso se evidenciaron a su vez, similitudes con resultados obtenidos en otros estudios latinoamericanos, en relación al nivel de percepción de cuidado humanizado otorgado por enfermeras(os) que presentaron los pacientes hospitalizados ${ }^{19,41,42}$, demostrando las adecuadas propiedades de este instrumento modificado para la comprensión de la población chilena, mostrando su buen funcionamiento en un contexto cultural distinto. 
A su vez, la metodología aquí empleada, para este tipo de adaptaciones dentro del mismo idioma, pero bajo contextos cultures distintos, se pesquisó como escasamente empleada ${ }^{21}$, siendo más utilizadas y recurrentes las adaptaciones y validaciones de instrumentos en distintos idiomas $^{21-24}$, lo cual puede ser considerado como una dificultad en la realización de este trabajo, ya que se pesquisó escasa literatura sobre la metodología a aplicar para una adaptación sociolingüística y de lo encontrado, no existía un conceso al respecto. Por lo cual este estudio se considera un aporte para futuras investigaciones en que se requiera realizar este proceso ya que, a la luz de este estudio, se visualiza la importancia de realizar este tipo de trabajos y adaptaciones socioculturales, aun cuando se comparta el mismo idioma, ya que interpretación errónea producto de las interferencias en la interpretación del lenguaje, pueden afectar los resultados de las investigaciones.

De esta forma, este cuestionario adaptado y validado para ser aplicado en población chilena se trasforma en una herramienta que puede ser de suma utilidad para ser aplicado en diversos ámbitos del ambiente hospitalario en Chile y de esta forma medir un aspecto tan relevante en nuestros días, el cual está íntimamente ligado a la calidad de los cuidados de enfermería. Como limitación de este estudio se puede mencionar que no se abarcaron todos los tipos de validación existentes, por lo que esta investigación deja la oportunidad para que se realicen más estudios en este ámbito, a su vez la segunda fase de esta investigación, fue desarrollada en el marco de una prueba piloto, por lo cual la muestra fue escueta, motivo por el que se sugiere que en estudios posteriores se trabaje con muestras mayores, para así generalizar los resultados de manera fehaciente.

\section{CONCLUSIONES}

La humanización del cuidado es un concepto amplio, que involucra múltiples aspectos necesarios para brindar un cuidado ético, basado en el respeto por la dignidad humana. En este escenario y bajo la relevancia que tiene el concepto de calidad en la atención en salud, es que se hace necesario contar con instrumentos confiables y veraces que permitan evaluar este aspecto trascendental del cuidado entregado por enfermería. De esta forma los resultados de esta investigación son útiles para la práctica de enfermería, ya que en primer lugar entrega una metodología de trabajo poco usada para la adaptación sociolingüística de instrumentos en el mismo idioma, evidenciando la importancia de realizar este proceso por el impacto que tiene las implicancias culturales en la forma de expresión y comprensión del lenguaje, lo cual puede afectar profundamente el resultado de una investigación. A su vez, este estudio entrega una herramienta que puede ser aplicada en otros estudios, para ir profundizando el análisis y medición del cuidado humanizado.

Estos resultados permitirán que se sigan realizando investigaciones en esta temática e ir avanzando en políticas y estrategias para otorgar una atención más humana y ética a las personas, atención centrada en el trato digno y que genere, a su vez, un cuidado seguro y de calidad para todos los usuarios y sus familias. 
Conflicto de intereses: Los autores declaran no tener ningún conflicto de intereses.

\section{REFERENCIAS}

1. Leite R, Brito E, Silva L, Palha P, Ventura C. Access to healthcare information and comprehensive care: perceptions of users of a public service. Interface (Botucatu). 2014; 18(51): 661-72. http://dx.doi.org/10.1590/1807-57622013.0653

2. Lopera MA, Forero C, Paiva LE, Cuartas VM. El quehacer cotidiano de la enfermera significa soportar la carga. Rev Cuid. 2016; 7(2): 1262-70. http://dx.doi.org/10.15649/cuidarte.v7i2.320

3. Arredondo-González C, Siles-González J. Tecnología y Humanización de los Cuidados: Una mirada desde la Teoría de las Relaciones Interpersonales. Index Enferm. 2009; 18(1): 32-6. https://dx.doi.org/10.4321/s1132-12962009000100007

4. Corbani N, Brêtas A, Matheus M. Humanização do cuidado de enfermagem: o que é isso? Rev Bras Enferm. 2009; 62(3): 349-54. http://dx.doi.org/10.1590/S0034-71672009000300003

5. Correa ML. La humanización de la atención en los servicios de salud: un asunto de cuidado. Rev Cuid. 2016; 7(1): 1227-31. http://dx.doi.org/10.15649/cuidarte. $\mathrm{v} 7 \mathrm{i} 1.300$

6. Alonso J. Cuidados paliativos: entre la humanización y la medicalización del final de la vida. Ciência \& Saúde Coletiva. 2013; 18(9): 2541-8.

http://dx.doi.org/10.1590/S1413-81232013000900008

7. Grísales-Naranjo L, Arias-Valencia M. Humanized care; the case of patients subjected to chemotherapy. Invest Educ Enferm. 2013; 31(3): 364-76.

8. Miqueletto M, Silva L, Figueira CB, Santos MR, Szylit R, Ichikawa CRF. Espiritualidade de famílias com um ente querido em situação de final de vida. Rev Cuid. 2017; $8(2)$ : $1616-27$. http://dx.doi.org/10.15649/cuidarte.v8i2.391

9. Freitas J, Silva A, Minamisava R, Bezerra A, Sousa Maiana R. Calidad de los cuidados de enfermería y satisfacción del paciente atendido en un hospital de enseñanza. Rev. Latino-Am. Enfermagem. 2014; 22(3): 454-60. http://dx.doi.org/10.1590/0104-1169.3241.2437

10. Watson J. The philosofhy and science of caring. $1^{\circ}$ ed. Colorado. University Press of Colorado; 2008.

11. Urra M, Jana A, García M. Algunos aspectos esenciales del pensamiento de Jean Watson y su teoría de cuidados transpersonales. Cienc enferm. 2011; 17(3): 11-22. http://dx.doi.org/10.4067/S0717-95532011000300002

12. Watson J. Assessing and measuring caring in nursing and health science. $1^{\circ}$ ed. New York. springer publishing company, Inc; 2002.
13. Morales-Castillo F, Hernández-Cruz M, MoralesRodríguez M, Landeros-Olvera E. Validación y estandarización del instrumento: Evaluación de los comportamientos de cuidado otorgado en enfermeras mexicanas. Enferm Universitaria. 2016; 13(1): 3-11. https://doi.org/10.1016/j.reu.2015.11.005

14. Poblete-Troncoso M, Valenzuela-Suazo S, Merino J. Validación de dos escalas utilizadas en la medición del cuidado humano transpersonal basadas en la Teoría de Jean Watson. Aquichan. 2012; 12(1): 8-21. https://doi.org/10.5294/aqui.2012.12.1.1

15. Reina N, Vargas R. Validez de contenido y validez facial del instrumento "Percepción de comportamientos de cuidado humanizado". Av. enferm. 2008; 26(2): 71-9.

16. González-Hernández O. Validez y confiabilidad del instrumento "Percepción de comportamientos de cuidado humanizado de enfermería PCHE $3^{\mathrm{a}}$ versión". Aquichan. 2015; 15(3): 381-92. https://doi.org/10.5294/aqui.2015.15.3.6

17. Coseriu E, Pastene F, Katabek J. Diez tesis a propósito de la esencia del lenguaje y del significado. Literatura y lingüística. 2006; (17): 327-31. http://dx.doi.org/10.4067/S0716-58112006000100018

18. Luis C. Lo arbitrario del lenguaje y el lugar de la cultura. Pro-Posições. 2008; 19(1): 189-201. http://dx.doi.org/10.1590/S0103-73072008000100019

19. Rivera L, Triana A. Percepción de comportamientos de cuidado humanizado de enfermería en la Clínica del Country / Perception of behaviors of humanized nursing care at Clínica del Country. Av. enferm. 2007; 25(1): 56-68.

20. Torres T, Camargo B, Bousfield A. Estereótipos sociais do idoso para diferentes grupos etários. Psic. Teor. e Pesq. 2016; 32(1): 209-18.

http://dx.doi.org/10.1590/0102-37722016012114209218

21. Aguilar L, Castellanos M. Adaptación Lingüístico-Cultural de una Escala Argentina de Perfeccionismo Infantil al Contexto Venezolano. Revista Electrónica de Metodología Aplicada. 2016; 21(2):1-32.

https://doi.org/10.17811/rema.21.2.2016.1-32

22. Rivero E, González L, Bayarre H. Validación y adaptación lingüística-cultural del Instrumento World Health Organization Quality of Live Bref (WHOQOL) en mujeres de edad mediana. Párraga, La Habana. 2013-2014. Rev Cubana Enfermer. 2017; 32(4): 126-39.

23. Ramada-Rodilla JM, Serra-Pujadas C, Delclós-Clanchet LG. Adaptación cultural y validación de cuestionarios de salud: revisión y recomendaciones metodológicas. Salud Pública Méx. 2013; 55(1): 57-66. https://doi.org/10.1590/S0036-36342013000100009

24. Congost-Maestre N. La Validación Lingüística en los Principales Cuestionarios de Salud y Calidad de Vida. Lebende Sprachen. 2015; 60(2): 307-25. http://dx.doi.org/10.1515/les-2015-0014

25. Oviedo H, Campo-Arias A. Aproximación al uso del coefciente alfa de Cronbach. Rev. Colomb. Psiquiatr. 2005; 34(4): 572-80. 
26. Hernández R, Fernández $\mathbf{C}$, Baptista $\mathbf{P}$. Recolección de los datos. En: Hernández R, Fernández C, Baptista $\mathrm{P}$. Metodología de la Investigación. $3^{\mathrm{a}}$ edición. México: $M c$ Graw Hill. 2003; 342-482.

27. Cardona-Arias J, Ospina-Franco L, Eljadue-Alzamora A. Validez discriminante, convergente/divergente, fiabilidad y consistencia interna, del whoqol-bref y el mossf-36 en adultos sanos de un municipio colombiano. Rev. Fac. Nac. Salud Pública. 2015; 33(1): 50-7.

28. Hermosilla A, Mendoza R, Contreras S. Instrumento para valoración del cuidado humanizado brindado por profesionales de enfermería a personas hospitalizadas. Index Enferm. 2016; 25 ( 4 ): 273-7.

29. Sáez LG. El léxico del dialecto chileno: Diccionario de uso del español de Chile DUECh. Estud. filol. 2012; (49): $137-$ 55. http://dx.doi.org/10.4067/S007117132012000100009

30. Sadowsky S, Martínez R. 2012. Lista de Frecuencias de Palabras del Castellano de Chile (Lifcach). Versión 2.0. Base de datos electrónica. Disponible en:

http://sadowsky.cl/lifcach.html

31. Oyanedel M, Samaniego J. Notas para un nuevo perfil lingüístico del español de Santiago de Chile. Boletín de Filología. 1998; 37(2): 899-913.

32. Arús J. Perspectiva sistémico-funcional de los usos de 'se' en español. Revista Signos. 2006; 39(61): 131-59. http://dx.doi.org/10.4067/S0718-09342006000200001

33. Grande F. La cortesía verbal como reguladora de las interacciones verbales. ASELE Actas XVI. 2005; 332-42.

34. Chile. Ley $\mathrm{N}^{\circ} 20.584$ que regula los derechos y deberes que tienen las personas en relación con acciones vinculadas a su atención en salud. Disponible en:

http://www.leychile.cl/Navegar?idNorma $=1039348$

35. Moreno-Fergusson M. Humanización del cuidado: una meta enraizada en la esencia de enfermería. Aquichan. 2013; 13(2): 146-7.

36. Calderon D, Verdi M. Cogestão e processo de intervenção de apoiadores da Política Nacional de Humanização (PNH). Comunicação saúde educação. 2014; 8(1): 859-70. http://dx.doi.org/10.1590/1807-57622013.0960

37. Congreso de Colombia. Ley 911 del 2004. Diario Oficial 45.693

38. Chahín-Pinzón N, Lorenzo-Seva U, Vigil-Colet A. Características psicométricas de la adaptación colombiana del Cuestionario de Agresividad de Buss y Perry en una muestra de preadolescentes y adolescentes de Bucaramanga. Universitas Psychologica. 2012; 11(3): 979-88.

39. Barbero MI, Vila E, Holgado F. La adaptación de los tests en estudios comparativos interculturales. Acción Psicológica. 2008; 5(2): 7-16 https://doi.org/10.5944/ap.5.2.454

40. Ferreira M, Haas V, Dantas R, Felix M, Galvão C. Cultural adaptation and validation of an instrument on barriers for the use of research results. Rev. Latino-Am. Enfermagem. 2017; 25: 28-52.

http://dx.doi.org/10.1590/1518-8345.1652.2852
41. Bautista-Rodríguez L, Parra-Carrillo E, Arias-Torres K, Parada-Ortiz K, Ascanio-Meza K, VillamarinCapacho M, et al. Percepción de los comportamientos de cuidado humanizado en los usuarios hospitalizados en una institución de salud de $3^{\circ}$ y $4^{\circ}$ nivel de atención. Rev. cienc. cuidad. 2015; 12(1): 105-18.

https://doi.org/10.22463/17949831.331

42. Romero E, Contreras I, Moncada A. Relación entre cuidado humanizado por enfermería con la hospitalización de pacientes. Hacia promoc. salud. 2016; 21(1): 26-36. https://doi.org/10.17151/hpsal.2016.21.1.3 